\title{
Serum Apolipoprotein A-Iv Level in HCV Related Liver Diseases
}

\author{
Authors \\ Ibrahim E.H. ${ }^{*}$, Elyamany A.S. ${ }^{*}$, Dwedar F.I. ${ }^{* *}$, Aly E.F. ${ }^{*}$ \\ *Department of Internal Medicine, **Department of Biochemistry, Faculty of Medicine, \\ University of Alexandria, Egypt
}

\begin{abstract}
Background: Hepatitis $C$ virus $(\mathrm{HCV})$ is a globally prevalent pathogen and chronic infection eventually causes cirrhosis leading to hepatocellular carcinoma (HCC) and ultimately death. Accurate assessment of liver fibrosis has become increasingly important as it allows for individualized management. Liver plays a key role in the metabolism of plasma apolipoproteins. Apolipoprotein A-IV (apoA-IV) is a component of intestinally derived, triglyceride-rich lipoproteins.

Objective: The present work was designed to study the level of apo A-IV in HCV related liver diseases.

Methods: Sixty treatment-naive patients with HCV related liver disease were enrolled in the study. They were classified as follow: twenty patients with chronic HCV infection (stage of fibrosis $\leq$ F3), twenty patients with $H C V$ related liver cirrhosis, twenty patients with $\mathrm{HCV}$ related $\mathrm{HCC}$ and ten normal subjects as control. Serum level of apoA-IV was measured using enzyme linked immunosorbant assay (ELISA). Liver biopsies from patients with chronic HCV were examined to assess histological activity grade and fibrosis stage according to METAVIR scoring system and triphasic CT for HCC patients was performed.

Results: Apo A-IV level was significantly lower in liver cirrhosis than chronic hepatitis patients with a cutoff point of $<36.4$ ( $p<0.001)$. The receiver operating characteristic $(R O C)$ curve showed an area under the curve (AUC) of 0.990 ( $P<0.001$ ), a sensitivity of $100 \%$ and a specificity of $90 \%$ for diagnosis of cirrhosis. Also, it was significantly lower in liver cirrhosis than HCC patients with a cutoff point $\leq 25.1$ ( $p<0.001$ ). The ROC showed AUC of 0.876, a sensitivity of $80.0 \%$ and a specificity of $93.33 \%$ for diagnosis of HCC.

Conclusion: Serum apo A-IV showed bi-modal elevation in HCV related liver diseases and could be used as a noninvasive marker for liver cirrhosis in patients with chronic HCV infection and could be a promising marker for early detection of HCC in HCV hepatic cirrhosis.

Key words: (Apolipoprotein A-IV, Hepatitis C Virus, Liver cirrhosis, Hepatocellular carcinoma)
\end{abstract}

\section{INTRODUCTION}

Hepatitis $\mathrm{C}$ virus continues to be a major cause of concern and a huge burden on public health systems worldwide. The WHO estimates that a minimum of 3 per cent of the world's population is chronically infected with HCV. (1) However, within this chronically infected population the disease outcomes 
vary, it can be mild or severe and can lead to scar tissue formation. Chronic infection eventually causes cirrhosis leading to HCC and ultimately death. ${ }^{(2)}$ Globally, at least one third of HCC cases are attributed to $\mathrm{HCV}$ infection and 350,000 people die from HCV related diseases per year. ${ }^{(3)}$ With the advancement in the treatment of patients with chronic liver diseases, the accurate assessment of liver fibrosis has become increasingly important as it allows for individualized management. The lack of accurate, reproducible and easily applied methods for assessment of hepatic fibrosis has been the major limitation for both the clinical management and research in liver diseases. ${ }^{(4)}$ For the past 50 years; liver biopsy has been considered to be the gold standard for staging of liver fibrosis. However, many recent studies clearly highlight several crucial drawbacks of liver biopsy. ${ }^{(5)}$ In recent years, interest in identifying and describing liver fibrosis by using non invasive surrogate markers has been on the rise. (6)

Under normal physiological conditions, liver ensures homeostasis of lipid and lipoprotein metabolism. Hepatic cellular damage and HCC impairs these processes, leading to alterations in plasma lipid and lipoprotein patterns. ${ }^{(7)}$ Understanding the tight association between HCV life cycle and lipoprotein metabolism is very important because $\mathrm{HCV}$ infection is a unique model wherein the virus causes chronic infection while coexisting with the host and simultaneously taking over the host's metabolism. ${ }^{(8)}$ In addition, the anomalous lipid metabolism caused by HCV infection may lead to liver injury and hepatocarcinogenesis. ${ }^{(9)}$ However, clinical studies have indicated that HCV induced over expression of lipogenic genes may exert a strong influence on inflammation and fibrosis progression of the infected liver, rather than causing the lipid accumulation observed in hepatic steatosis. ${ }^{(10)}$ Mature apo A-IV is a 377 amino acids peptide and contains about $6 \%$ carbohydrate. It is present in plasma in various isoforms. Its gene is located on chromosome 11. It is synthesized in the intestine. ${ }^{(1)}$

\section{SUBJECTS AND METHODS}

This study was conducted on 60 patients with $\mathrm{HCV}$ related liver disease. The patients were classified into three groups: Twenty patients with chronic hepatitis $\mathrm{C}(\mathrm{CHC})$ who were diagnosed by presence of circulating anti $\mathrm{HCV}$ antibody and histopathological finding consistent with chronic hepatitis (stage of fibrosis $\leq$ F3), twenty patients with HCV related liver cirrhosis (based on clinical, laboratory and/or radiological finding of cirrhosis), twenty patients with HCV related HCC (based on having focal hepatic lesion(s) in a background of cirrhosis evidenced by triphasic CT and the characteristic criteria of HCC) and ten normal individual were enrolled in the control arm. The human apo A-IV ELISA kit (Wuhan EIAab Science Co., Ltd. optics valley, Wuhan, China) was measured in the serum of all enrolled cases. $^{(12)}$

Patients were excluded from the study if they had HBV infection; concomitant schistosomiasis; history of alcohol consumption; other known causes of chronic liver disease; cardiac, respiratory or renal diseases; any kind of malignancy or known chronic diseases such as diabetes mellitus, or connective tissue disorders. Patients who had received anti-viral or immunomodulatory therapy were also excluded. The study was conducted in accordance with the provisions of the Declaration of Helsinki and Good Clinical Practice guidelines. An informed consent was obtained from all subjects prior to procedures initiation.

Once admitted into the study, the base line assessment included: age, gender, symptoms and signs of chronic liver disease, liver and spleen size, serum albumin, serum bilirubin, serum aspartate and alanine aminotransferases (AST and ALT respectively), serum gamma glutamyl transpeptidase (GGT), prothrombin time, international normalized ratio and serum creatinine. HCV antibodies, hepatitis B surface antigen and hepatitis B core antibody using ELISA and HCV RNA levels in serum using real time polymerase chain reaction assay. Abdominal 
ultrasonographic examination was used to assess: liver echo-pattern and presence of hepatomegaly. While triphasic CT was employed to assess focal hepatic lesions to establish HCC diagnosis.

\section{Histopathological examination:}

Core liver biopsies were obtained from all patients with CHC. All specimens were fixed in a $10 \%$ formalin solution and embedded in paraffin. Tissue sections were stained with: HematoxylinEosin and Trichrome Stains for assessment of necroinflammation grade and fibrosis stage according to the METAVIR scoring system composed of a two-letter and two-number coding system: ${ }^{(13)} \mathrm{A}=$ Histological activity was graded as $\mathrm{A} 0=$ no activity, $\mathrm{A} 1=$ mild activity, $\mathrm{A} 2=$ moderate activity and $\mathrm{A} 3=$ severe activity. $\mathrm{F}=$ Fibrosis stage was defined as F0 = no fibrosis, F1 = portal fibrosis without septa, F2 = portal fibrosis with rare septa, F3 = numerous septa without cirrhosis and F4 $=$ cirrhosis.

\section{Statistical analysis of the data ${ }^{(14)}$}

Data were fed to the computer and analyzed using IBM SPSS software package version 20.0. ${ }^{(15)}$ Qualitative data were described using number and percent. Quantitative data were described using range (minimum and maximum) mean, standard deviation and median. Comparison between different groups regarding categorical variables was tested using Chi-square test. When more than $20 \%$ of the cells showed count less than 5, correction for chi-square was conducted using Monte Carlo correction. The distributions of quantitative variables were tested for normality using Kolmogorov-Smirnov test, Shapiro-Wilk test and D'Agstino test, also Histogram and QQ plot were used for vision test. Normal data distribution entailed parametric tests while nonparametric tests were applied in presence of abnormally distributed data. For normally distributed data, comparison between the different groups was done using F-test (ANOVA) and Post Hoc test (Tukey) while for abnormally distributed data, comparison was done using Kruskal Wallis test and pair wise comparison was assessed using Mann-Whitney test. Significance of the obtained results was judged at the $5 \%$ level.

\section{RESULTS}

Characteristics of studied patients are presented in tables I. Table II showed the statistical comparisons between patients with $\mathrm{CHC}$, cirrhosis, HCC and healthy subjects as regards the serum level of apo A-IV. Apo A-IV level was significantly lower in liver cirrhosis than chronic hepatitis patients with a cutoff point of $<36.4$ $(\mathrm{p}<0.001)$. As shown in figure 1 , the ROC curve showed an AUC of $0.990(\mathrm{P}<0.001)$, a sensitivity of $100 \%$ and a specificity of $90 \%$ for diagnosis of cirrhosis. Also, it was significantly lower in liver cirrhosis than HCC patients with a cutoff point $\leq 25.1 \quad(\mathrm{p}<0.001)$. The ROC showed AUC of 0.876 , a sensitivity of $80.0 \%$ and a specificity of 93.33\% for diagnosis of HCC. (as shown in figure 2) There was no significant correlation between apo A-IV level and age, gender, liver profile tests, AFP, lipid profile, CRP, hepatic histopathological changes nor the BCLC. 
Table I: Characteristics of studied patients

\begin{tabular}{|c|c|c|c|}
\hline Gender & 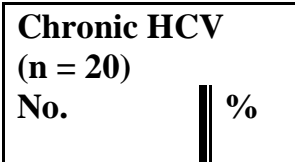 & 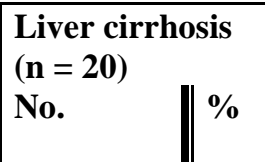 & \begin{tabular}{|l||l|} 
HCC & \\
$(\mathbf{n}=20)$ & \\
No. & $\%$
\end{tabular} \\
\hline Male & \begin{tabular}{l||l}
8 & 40.0
\end{tabular} & \begin{tabular}{l||l}
12 & 60.0
\end{tabular} & 55.0 \\
\hline Female & \begin{tabular}{l||l}
12 & 60.0
\end{tabular} & \begin{tabular}{l||l}
8 & 40.0
\end{tabular} & 45.0 \\
\hline Age (years) & $44.15 \pm 6.33$ & $59.05 \pm 5.69$ & $62.85 \pm 4.51$ \\
\hline $\operatorname{ALT}(\mathbf{U} / \mathbf{L})$ & $38.35 \pm 15.97$ & $49.65 \pm 22.97$ & $48.45 \pm 17.73$ \\
\hline AST (U/L) & $42.35 \pm 14.43$ & $63.95 \pm 25.89$ & $58.65 \pm 25.04$ \\
\hline Serum albumin $(g / d L)$ & $3.75 \pm 0.30$ & $2.67 \pm 0.48$ & $2.71 \pm 0.44$ \\
\hline Total Bilirubin (mg/dL) & $0.88 \pm 0.33$ & $2.22 \pm 1.61$ & $2.45 \pm 1.67$ \\
\hline Direct Bilirubin (mg/dL) & $0.34 \pm 0.24$ & $1.36 \pm 1.14$ & $1.72 \pm 1.46$ \\
\hline GGT (U/L) & $24.85 \pm 7.52$ & $23.45 \pm 7.86$ & $25.60 \pm 9.28$ \\
\hline Prothrombin activity (\%) & $93.12 \pm 4.72$ & $58.03 \pm 12.96$ & $63.83 \pm 13.56$ \\
\hline Alpha feto protein $(\mathrm{ng} / \mathrm{mL})$ & $4.42 \pm 1.97$ & $6.46 \pm 2.36$ & $1071.38 \pm 1970.87$ \\
\hline $\operatorname{HbA1C}(\%)$ & $5.89 \pm 0.36$ & $5.89 \pm 0.36$ & $5.80 \pm 0.45$ \\
\hline$C$ reactive protein $(\mathrm{mg} / \mathrm{L})$ & $2.48 \pm 1.62$ & $2.96 \pm 1.60$ & $2.76 \pm 2.20$ \\
\hline Total cholesterol (mg/dL) & $125.60 \pm 21.04$ & $124.90 \pm 18.40$ & $141.90 \pm 31.58$ \\
\hline Tri glyceride (mg/dL) & $104.0 \pm 25.33$ & $102.25 \pm 22.24$ & $89.45 \pm 22.06$ \\
\hline HDL (mg/dL) & $25.45 \pm 7.79$ & $25.65 \pm 7.67$ & $26.50 \pm 7.03$ \\
\hline LDL (mg/dL) & $55.60 \pm 19.85$ & $51.40 \pm 25.11$ & $70.20 \pm 30.99$ \\
\hline
\end{tabular}

ALT: alanin aminotransferase, AST: aspartate aminotransferase, GGT: gamma-glutamyltransferase, HbA1c:glycated hemoglobin, HDL : High density lipoprotein , LDL : Low density lipoprotein

Table II: Comparison between the four groups according to apolipoprotein A-IV serum level

\begin{tabular}{|c|c|c|c|c|c|c|}
\hline & \begin{tabular}{|l} 
Chronic HCV \\
$(\mathbf{n}=20)$
\end{tabular} & $\begin{array}{l}\text { Liver cirrhosis } \\
(\mathbf{n}=\mathbf{2 0})\end{array}$ & $\begin{array}{l}\text { HCC } \\
(\mathbf{n}=\mathbf{2 0})\end{array}$ & $\begin{array}{l}\text { Control } \\
(\mathbf{n}=10)\end{array}$ & ${ }^{\mathrm{Kw}} \chi^{2}$ & $\mathbf{P}$ \\
\hline $\begin{array}{l}\text { Apolipoprotein A- } \\
\text { IV(ng/ml) } \\
\text { Min. - Max. } \\
\text { Mean } \pm \text { SD. } \\
\text { Median } \\
\end{array}$ & $\begin{array}{l}37.80-98.70 \\
68.19 \pm 17.63 \\
71.05\end{array}$ & $\begin{array}{l}15.50-46.10 \\
25.01 \pm 7.91 \\
23.10\end{array}$ & $\begin{array}{l}24.10-69.80 \\
37.59 \pm 11.10 \\
36.90\end{array}$ & $\begin{array}{l}25.20-56.20 \\
39.13 \pm 8.97 \\
38.65\end{array}$ & $46.847^{*}$ & $<0.001^{*}$ \\
\hline \begin{tabular}{|l|} 
P Cont. \\
Sig.bet. Grps. \\
\end{tabular} & $\begin{array}{l}<0.001^{*} \\
\mathrm{p}_{1}<0.001^{*}, \mathrm{p}_{2}<0\end{array}$ & $\begin{array}{l}<0.001^{*} \\
01^{*}, \mathrm{p}_{3}<0.001^{*}\end{array}$ & 0.428 & & & \\
\hline
\end{tabular}

KW: Kruskal Wallis test, grps was done using Mann Whitney test

$\mathrm{p}_{\text {cont }}$ : $\mathrm{p}$ value for comparing between group control and each other group

$\mathrm{p}_{1}$ : $\mathrm{p}$ value for comparing between chronic $\mathrm{HCV}$ and liver cirrhosis

$\mathrm{p}_{2}$ : $\mathrm{p}$ value for comparing between chronic $\mathrm{HCV}$ and $\mathrm{HCC}$

$\mathrm{p}_{3}$ : $\mathrm{p}$ value for comparing between liver cirrhosis and $\mathrm{HCC}$

*: Statistically significant at $\mathrm{p} \leq 0.05$

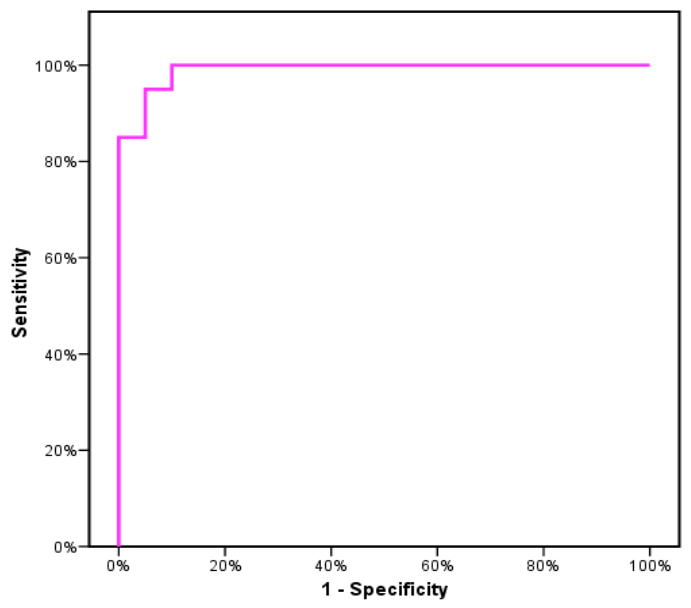

Figure (1): ROC curve for Apolipoprotein A-IV between chronic HCV and cirrhotic patients 


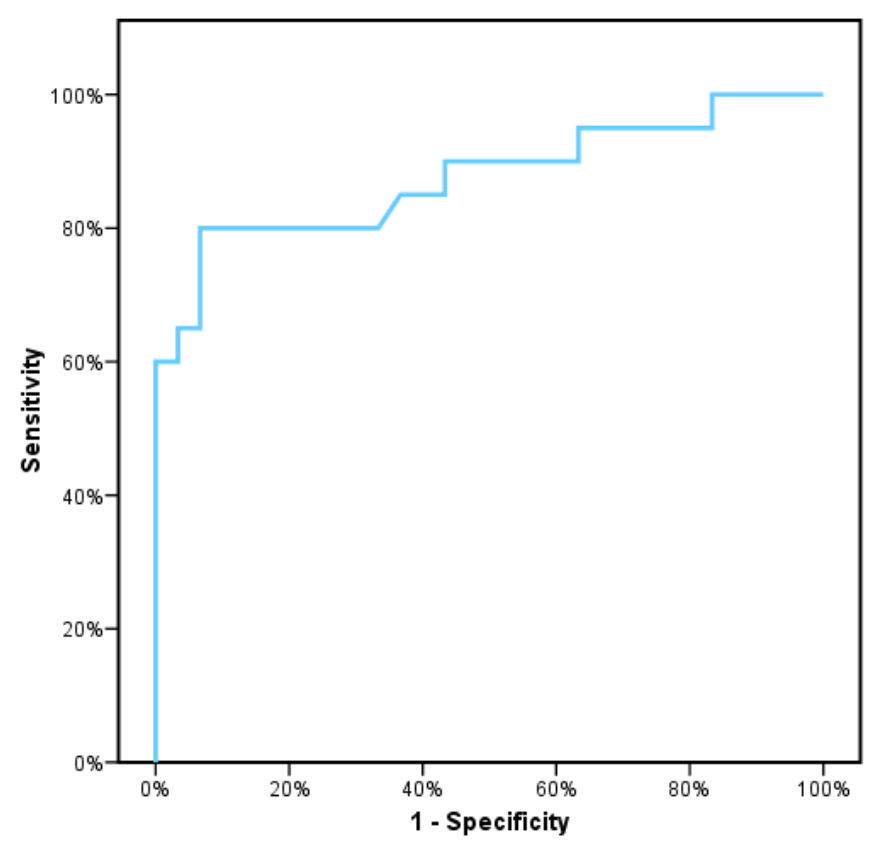

Figure (2): ROC curve for Apolipoprotein A-IV between HCV related cirrhosis and HCC

\section{DISCUSSION}

In the present study, apo A-IV level greatly reduced in the liver cirrhosis patients, compared with the chronic hepatitis patients and reduced level of apo A-IV in liver cirrhosis group compared to HCC group. In agreement with these results, Seishima et al, found that there was a decreased intestinal apo A-IV and its mRNA levels in a carbon tetrachloride (CCl4)-induced cirrhosis rat model. This may be related to the decreased ability to absorb fat in cirrhosis. (16) Also, Pleguezuelo et al, compared its level in plasma between cirrhotic patients with and without HCC. They found that apo A-IV was significantly higher in patients without HCC. Their study revealed that levels of apoA-1 and apoA-IV were the only factors independently associated with HCC. Interestingly, apoA-IV was associated with lower risk of HCC. ROC curve for this equation using a cut-off level of 0.35 showed an area under the ROC of 0.91 ; with $89 \%$ sensitivity and $91 \%$ specificity for diagnosis of HCC. ${ }^{(17)}$ They conducted their study on patients with liver cirrhosis secondary to (HBV, HCV and alcoholic liver disease) in contrast to our study which was conducted on $\mathrm{HCV}$ related cirrhosis and HCC.
Using two-dimensional polyacrylamide gel electrophoresis, Sugimoto et al attempted to isolate novel specific biomarker in the sera of patients with chronic hepatitis, cirrhosis and HCC. One of the differentially expressed proteins was apo A-IV isoform. No change was found in the expression pattern of this isoform in either normal or chronic hepatitis sera, whereas a significant reduction in the expression level of this protein was observed in the sera of LC and HCC patients. (12)

Metabolic syndrome has closely linked to the development of human HCC. By using the HBV $\mathrm{X}(\mathrm{HBx})$ transgenic mouse model, Teng et al studied the dynamic evolution of serum and liver profiles of lipids and global cDNA expression at different stages of $\mathrm{HBx}$ tumorigenesis. They observed that the lipid (triglycerides, cholesterol, and fatty acids) profiles revealed a biphasic response pattern during the progression of $\mathrm{HBx}$ tumorigenesis: a small peak at early phase and a large peak or terminal switch at the tumor phase. By analyzing cDNA microarray data, the early peak correlated to the oxidative stress and proinflammatory response, which then resolved at the middle phase and were followed by the terminal metabolic switch in the tumor tissues. Five lipid 
metabolism-related genes, the arachidonate 5lipoxygenase, lipoprotein lipase, fatty acid binding protein 4, 1-acylglycerol-3-phosphate Oacyltransferase 9, and apo A-IV were identified to be significantly activated in HBx transgenic HCCs and further validated in human HBV-related HCCs. Inhibition of these lipid genes could reverse the effect of $\mathrm{HBx}$ on lipid biosynthesis and suppress HBx-induced cell proliferation in vitro. These results support the concept that metabolic syndrome plays an important role in $\mathrm{HBV}$ tumorigenesis. The dysregulation of lipid metabolic genes may predict the disease progression to $\mathrm{HCC}$ in chronic hepatitis B patients. ${ }^{(18)}$

Level of apo A-IV is altered in HCC patient because lipids and lipoprotein metabolism could be regulated by cytokines. For instance, interleukin-6 (IL-6), tumor necrosis factor (TNF$\alpha$ ), IL-1 may inhibit TG synthesis. ${ }^{(19)}$ Tumor cells are known to produce large amounts of proinflammatory cytokines known to suppress plasma TG levels. Argiles, et al, reported that IL1 profoundly affects lipid metabolism by delaying intestinal absorption and decreasing tissue uptake. (20) IL-1 and IL-6 significantly decreased microsomal triglyceride transfer protein mRNA levels in HepG2 cells. ${ }^{(21)}$ Apo A-IV has been also incriminated in other types of cancers. Zervos et al found that apo A-IV gene was up-regulated in familial pancreatic cancer PCA. ${ }^{(22)}$

Li et al, ${ }^{(23)}$ studied the influence of apoA-IV on the secretion of cytokines stimulated by lipopolysaccharide (LPS) in the cells. The macrophage RAW264.7 cells were pre-treated with apo A-IV $(20 \mu \mathrm{g} / \mathrm{ml})$ for one hour, followed with LPS $(500 \mathrm{ng} / \mathrm{ml})$ for $20 \mathrm{~h}$. The culture medium was collected and RayBio® Mouse Inflammation Antibody Array G Series I was used to measure the cytokines. ApoA-IV was found to reduce Fas ligand, Fractalkine and Eotaxin-2. Both apo A-IV and LPS increased the cytokines GCSF, IL-2, IL-3, IL-4, IL-6, IL-9, IL-12P40/P70, GCSF, TIMP-1, LIX, SDP-1, TCA-3, TECK, TIMP-2, sTNF-RI, STNF-RII, GM-CSF, RANTES, I-TAC, KC,
MCP1,TNF- $\alpha$, TECK, IL-17 and Leptin. Notably, TNF- $\alpha$ and TECK induced by LPS were significantly inhibited by apo A-IV. Interestingly, apo A-IV enhanced IL-10 and IL- $1 \alpha$ by itself and that induced by LPS.

This significant change in apo A-IV serum level in liver disease could be due to the peculiar relationship between $\mathrm{HCV}$ infection and lipids as $\mathrm{HCV}$ virions circulate in serum bound to lipoproteins and lipids have been shown to modulate (and, indeed, are essential to) the HCV life cycle. As a result, lipid metabolism is overall altered, conferring an idiosyncratic profile to $\mathrm{HCV}$ infection. ${ }^{(24)}$ Most Apo, lipids and lipoproteins, are synthesized in the liver. Thus hepatocellular injury or chronic liver diseases including HCC may result in abnormal pattern of these molecules in plasma. ${ }^{(25)}$ Although the precise physiological function of apoA-IV has yet to be deduced, its altered level in chronic hepatitis group compared to the control group may be due to its anti inflammatory anti oxidant role which can be proved through several studies which had demonstrated its anti atherogenic role. Wong et al found that apo A-IV was found to be protected against atherosclerosis without an increase in HDL concentration and displayed reduced oxidative markers. Apo A-IV was found to have accumulated in the damaged arterial wall of these animals thus acting as an antioxidant in situ. (26)

Recalde et al suggested that human apolipoprotein (h-apoA-IV) exerts its protective role by inhibiting the lipopolysaccharide (LPS)-induced stimulation of monocytes. The systemic proinflammatory response was less strong in LPS-stimulated human apolipoprotein A-IV in apoE-deficient mice( $h$ apoA-IV/E0) mice than in LPS-stimulated apoEdeficient (apoE0) mice. ${ }^{(27)}$ Also, Khovidhunkit et al suggested that apo A-IV and A-V are positive acute-phase proteins that increase in the serum during inflammation. They found that Interleukin6 increased apo A-IV and apo A-V mRNA levels in Hep3B cells ${ }^{(28)}$ Spaulding et al found that apo A-IV could limit the cellular apoptotic response to two different oxidant chemicals: tert-butyl 
hydroperoxide ( $\mathrm{TBH}$ ), a model hydroperoxide, and diamide, a cell-permeantthiol oxidant, A-IV resembles $\mathrm{N}$-acetyl cysteine ( NAC) in its protective ability $^{(29)}$

After low-number transplantation of islets of Langerhans into the liver of streptozotocindiabetic rats, the hepatocytes in the acini are exposed to a local hyperinsulinemia, whereas the remaining tissue is affected by hypoinsulinemia. In this model, insulin induces alterations that resemble preneoplastic foci of altered hepatocytes (FAH) and develop into hepatocellular tumors in later stages of carcinogenesis. In rodents, apo AIV is synthesized in the small intestine and the liver. Whereas intestinal production is mainly influenced by lipid intake and chylomicrone formation, little is known about mechanisms regulating hepatic A-IV synthesis. As it is known that insulin modulates lipoprotein metabolism in different ways, they investigated the effect of insulin on hepatocytic A-IV mRNA expression in this model. After Laser microdissection of FAH and quantitative RT-PCR (LightCycler), a 3.2 to 7.4-fold increase of A-IV mRNA in the $\mathrm{FAH}^{\left({ }^{(30)}\right.}$

\section{CONCLUSION}

Measurement of serum apo A-IV can be a useful parameter in the follow up of $\mathrm{HCV}$ patients. Serum apo A-IV could be considered as noninvasive marker for liver cirrhosis with cut off point $>36.4,100 \%$ sensitivity and $90 \%$ specificity. It could predict the presence of $\mathrm{HCC}$ in $\mathrm{HCV}$ infected patient with liver cirrhosis with cut off point $\leq 25.1$ with sensitivity $80.0 \%$ and specificity $93.33 \%$.

\section{REFERENCES}

1. Cooke GS, Lemoine M, Thursz M, Gore C, Swan T, Kamarulzaman A, et al. Viral hepatitis and the Global Burden of Disease: a need to regroup. J Viral Hepat2013;20:6001.
2. Mohd HK, Groeger J, Flaxman AD, Wiersma ST. Global epidemiology of hepatitis $\mathrm{C}$ virus infection: new estimates of age-specific antibody to HCV seroprevalence. Hepatol 2013; 57:1333-42.

3. HanafiahKM,GroegerJ,FlaxmanAD, WiersmaST. Global Epidemiology of Hepatitis C Virus Infection: New Estimates of Age-Specific Antibody to HCV Seroprevalence. Hepatol 2013; 57:1333-42.

4. Guerra J, Garenne M, Mohamed MK,Fontanet A. HCV burden of infection in Egypt: results from a nationwide survey. J Viral Hepat2012; 19:560-7.

5. Miller FD, Abu-Raddad LJ. Evidence of intense ongoing endemic transmission of hepatitis $\mathrm{C}$ virus in

Egypt.ProcNatlAcadSciUSA2010;10 7(33):14757-6.

6. Kong F, Pan Y, Chi X, Wang $\mathrm{X}$, Chen L, Lv J,etal. Factors Associated with Spontaneous Clearance of Hepatitis C Virus in Chinese Population. BioMed Research International2014; 1-6.

7. BengschB, ThimmeR, BlumHE. Role of Host Genetic Factors in the Outcome of Hepatitis C Virus Infection.Viruses 2009; 1:104-25.

8. Afzal M, Tahir S, Salman A, Baig T, Shafi T, Zaidi N, etal. Analysis of interleukin-10 gene polymorphisms and hepatitis C susceptibility in Pakistan. J Infect DevCtries 2011; 5(6):473-9.

9. MeraniS, PetrovicD, James I,Abha Chopra, Cooper D, FreitaE, et al. Effect of immune pressure on hepatitis $\mathrm{C}$ virus evolution: insights 
from a single-source outbreak. Hepatol 2011; 53:396-405.

10. Burdette D, Haskett A, Presser L, McRae S, Iqbal J, Waris G. Hepatitis $C$ virus activates interleukin- $1 \beta$ via caspase-1-inflammasome complex. J Gen Virol2012;93:235-46.

11. Negash AA, Ramos HJ, Crochet N, Lau DT, Doehle B, Papic N, et al. IL-1 $\beta$ production through the NLRP3 inflammasome by hepatic macrophages links hepatitis $\mathrm{C}$ virus infection with liver inflammation and disease. PLoSPathog 2013;9:e1003330.

12. Sugimoto K, Shiraki K, Takei Y, Ito M, Nobori T, Suzuki H, et al. Serum protein isoform profiles indicate the progression of hepatitis C virusinduced liver diseases. Intern J Mole Med 2013; 31(4):943-50.

13. Bedossa P, Poynard T. An algorithm for grading activity in chronic hepatitis C. the French METAVIR Cooperative Study Group.Hepatology 1996;24:289-93.

14. Kotz S, Balakrishnan N, Read CB, Vidakovic B. Encyclopedia of statistical sciences. 2nd ed. Hoboken, N.J. Wiley-Interscience; 2006.

15. Kirkpatrick LA, Feeney BC. A simple guide to IBM SPSS statistics for version 20.0. Student ed. Belmont, Calif. Wadsworth, Cengage Learning; 2013.

16. Seishima M, Usui T, Naganawa S, Nishimura M, Moriwaki H, Muto Y, et al. Reduction of intestinal apo AIV mRNA levels in the cirrhotic rat. J Gastroenterol Hepatol 1996;11(8):746-51.

17. Pleguezuelo M, Lopez-Sanchez LM, Rodriguez-Ariza A, Montero JL,
Briceno J, Ciria R, et al. Proteomic analysis for developing new biomarkers of hepatocellular carcinoma. World J Hepatol 2010; 2(3): 127-35.

18. Teng CF, Hsieh WC, Yang CW, Su HM, Tsai TF, Sung WC, et al. A biphasic response pattern of lipid metabolomics in the stage progression of hepatitis $\mathrm{B}$ virus $\mathrm{X}$ tumorigenesis. Mol Carcinog 2015; In Press

19. Michiel DF, Oppenheim JJ. Cytokines as positive and negative regulators of tumor promotion and progression.Semin Cancer Biol 1992; 3(1):3-15.

20. Argiles JM, Lopez-Soriano FJ, Evans RD, Williamson DH. Interleukin-1 and lipid metabolism in the rat. Biochem J 1989; 259(3):6738.

21. Navasa M, Gordon DA, Hariharan N, Jamil H, Shigenaga JK, Moser A, et al. Regulation of microsomal triglyceride transfer protein mRNA expression by endotoxin and cytokines. J Lipid Res 1998; 39(6):1220-30.

22. Zervos EE, Tanner SM, Osborne DA, Bloomston M, Rosemurgy AS, Ellison EC et al. Differential gene expression in patients genetically predisposed to pancreatic cancer. J Surg Res 2006; 135(2):31722.

23. Li X, Li Z, Yan J, Tso P. ApoA-IV Regulates Cytokine Responsiveness in LPS-challenged Macrophage Cells.FASEB J2015; 29: Suppl LB197. 
24. Negro F. Abnormalities of lipid metabolism in hepatitis $\mathrm{C}$ virus infection. Gut 2010;59(9):1279-87.

25. Jiang JT, Wu CP, Xu N, Zhang XG. Mechanisms and significance of lipoprotein (a) in hepatocellular carcinoma. Hepatobiliary Pancreat Dis Int 2009;8:25-8.

26. Wong WR, Stephens JW, Acharya J, Hurel SJ, Humphries SE, Talmud PJ. TheAPOA4T347S variant is associated with reduced plasma TAOS in subjects with diabetes mellitus and cardiovascular disease Journal of Lipid Research2004;45: 1565-71.

27. Recalde D, Ostos MA, Badell E, Garcia-Otin AL, Pidoux J, Castro G, et al. Human Apolipoprotein A-IV Reduces Secretion of Proinflammatory Cytokines and Atherosclerotic Effects of a Chronic Infection Mimicked by Lipopolysaccharide. Arteriosclerosis, Thrombosis, and Vascular Biology2004; 24: 756-61.

28. Khovidhunkita W, Duchateauc PN, Medzihradszkyd KF, Mosera AH, Naya-Vignec J, Shigenagaa JK, etal. Apolipoproteins A-IV and A-V are acute-phase proteins in mouse HDL. Atherosclerosis 2004; 176(1): $37-44$.

29. Spaulding HL, Saijo F, Turnage RH, Alexander JS, Aw TY, Kalogeris TJ. Apolipoprotein AIV attenuates oxidant-induced apoptosis in mitotic competent, undifferentiated cells by modulating intracellular glutathione redox balance. Am J Physiol Cell Physiol2006; 290 ( 1): 95-103.
30. Evert M, Schneider-Stock R, Dombrowski F. Apolipoprotein AIV mRNA Overexpression in Early Preneoplastic Hepatic Foci Induced by Low-number Pancreatic Islet Transplants in Streptozotocindiabetic Rats. Pathol Res Pract 2003;199(6):373-9. 